\title{
CORRECTION
}

\section{Correction to: A Step Toward Realizing Open Volumetric Air Receiver Based Systems in Desert Regions}

\author{
G. Singh $^{1}\left(\mathbb{D} \cdot\right.$ P. Dhurwe $^{1} \cdot$ R. Kumar ${ }^{1} \cdot$ L. Kumar ${ }^{1} \cdot$ N. Vaghela $^{1} \cdot$ L. Chandra $^{1,2}$
}

Published online: 8 March 2019

(c) Indian National Academy of Engineering 2019

\section{Correction to: INAE Letters \\ https://doi.org/10.1007/s41403-018-0055-y}

In the original publication, the format of Table 1 is incorrect due to improper spanning of the rows. The correct format for Table 1 is given below:

The original article has been corrected.

Table 1 Expressions for the pressure-drop estimation across an absorber

\begin{tabular}{|c|c|c|c|}
\hline Absorber pore type & Equation & Expression & \\
\hline \multirow[t]{3}{*}{ Foam } & Ergun (1952) & $\frac{\Delta p}{L}=\frac{150 \mu_{f}(1-\varepsilon)^{2} u_{f}}{d_{p}^{2} \varepsilon^{3}}+\frac{1.75 \rho_{f}(1-\varepsilon) u_{f}^{2}}{d_{p} \varepsilon^{3}}$ & \\
\hline & Innocentini et al. (1999) & $\frac{\Delta p}{L}=\frac{66.67 \mu_{f} u_{f}}{d_{p}^{2}}+\frac{1.17 \rho_{f} u_{f}^{2}}{d_{p} \varepsilon^{2}}$ & \\
\hline & Lacroix et al. (2007) & $\frac{\Delta p}{L}=\frac{66.67 \mu_{f}(1-\varepsilon)^{2} u_{f}}{d_{s}^{2} \varepsilon^{3}}+\frac{1.17 \rho_{f}(1-\varepsilon) u_{f}^{2}}{d_{s} \varepsilon^{3}}, d_{s}=\frac{d_{p}[4 / 3 \pi(1-\varepsilon)]^{1 / 2}}{1-[4 / 3 \pi(1-\varepsilon)]^{1 / 2}}$ & (1) \\
\hline \multirow[t]{4}{*}{ Straight } & $\begin{array}{l}\text { Hagen-Poiseuille (HP) + minor loss } \\
\text { (White 2015) }\end{array}$ & $\frac{\Delta p}{L}=\frac{32 \mu_{f} \mathrm{v}_{p}}{d_{p}^{2}}, \Delta p_{\text {minor }}=\frac{1}{2} \rho_{f} \mathrm{v}_{p}^{2}\left[\left(1-\frac{A_{n p}}{A_{p i p e}}\right)^{2}+0.42\left(1-\frac{A_{n p}}{A_{\text {pipe }}}\right)\right]$ & \\
\hline & Sherman (1992) & $\Delta p=\frac{32 \mu_{f} \mathrm{Lv}_{p}}{d_{p}^{2}}+m \frac{1}{2} \rho_{f} \mathrm{v}_{p}^{2}, m=2.28$ & \\
\hline & Holmes (1967) & $\Delta p=\frac{1}{2} \rho_{f} \mathrm{v}_{p}^{2}\left(K+K^{\prime} / \operatorname{Re}\right), K=2.24, K^{\prime}=38.3$ & \\
\hline & Astarita and Greco (1968) & $\Delta p=\frac{795}{\operatorname{Re}} \frac{\rho_{f} v_{p}^{2}}{2}$ for $\operatorname{Re} \leq 146,5.48 \frac{\rho_{f} v_{p}^{2}}{2}$ for $\operatorname{Re}>146$ & \\
\hline
\end{tabular}

The original article can be found online at https://doi.org/10.1007/ s41403-018-0055-y.

L. Chandra

chandra.mec@iitbhu.ac.in; dr.laltu.chandra@gmail.com

1 Department of Mechanical Engineering, Indian Institute of Technology Jodhpur, Rajasthan 342037, India

2 Centre for Solar Energy Technologies, Indian Institute of Technology Jodhpur, Rajasthan 342037, India 